\title{
Powering the Next Billion Devices with Wi-Fi
}

\author{
Vamsi Talla, Bryce Kellogg, Benjamin Ransford, Saman Naderiparizi, \\ Shyamnath Gollakota and Joshua R. Smith \\ University of Washington
}

\begin{abstract}
We present the first power over Wi-Fi system that delivers power to low-power sensors and devices and works with existing Wi-Fi chipsets. Specifically, we show that a ubiquitous part of wireless communication infrastructure, the Wi-Fi router, can provide far field wireless power without significantly compromising the network's communication performance. Building on our design, we prototype battery-free temperature and camera sensors that we power with Wi-Fi at ranges of 20 and 17 feet respectively. We also demonstrate the ability to wirelessly trickle-charge nickelmetal hydride and lithium-ion coin-cell batteries at distances of up to 28 feet. We deploy our system in six homes in a metropolitan area and show that it can successfully deliver power via Wi-Fi under real-world network conditions without significantly degrading network performance.
\end{abstract}

\section{INTRODUCTION}

In the late 19th century, Nikola Tesla dreamed of eliminating wires for both power and communication [37]. As of the early 21 st century, wireless communication is extremely well established-billions of people rely on it every day. Wireless power, however, has not been as successful. In recent years, near-field, short range schemes have gained traction for certain range-limited applications, like powering implanted medical devices [42] and recharging cars [12] and phones from power delivery mats $[11,19,26]$. More recently researchers have demonstrated the feasibility of powering sensors and devices in the far field using RF signals from TV [25,33] and cellular [31, 41] base stations. This is exciting, because in addition to enabling power delivery at farther distances, RF signals can simultaneously charge multiple sensors and devices due to their broadcast nature.

This paper shows that a ubiquitous part of wireless infrastructure, the Wi-Fi router, can provide far-field wireless

Permission to make digital or hard copies of all or part of this work for personal or classroom use is granted without fee provided that copies are not made or distributed for profit or commercial advantage and that copies bear this notice and the full citation on the first page. Copyrights for components of this work owned by others than ACM must be honored. Abstracting with credit is permitted. To copy otherwise, or republish, to post on servers or to redistribute to lists, requires prior specific permission and/or a fee. Request permissions from Permissions@acm.org.

CoNEXT'15, December 01-04, 2015, Heidelberg, Germany

(C) 2015 ACM. ISBN 978-1-4503-3412-9/15/12 $\$ 15.00$.

DOI: http://dx.doi.org/10.1145/2716281.2836089

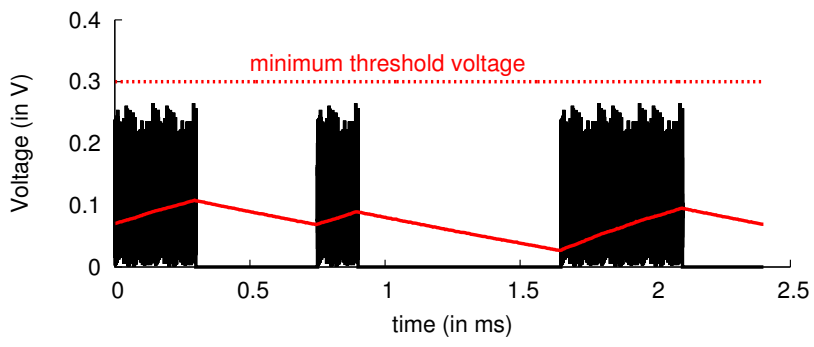

Figure 1-Key challenge with Wi-Fi power delivery. While the harvester can gather power during Wi-Fi transmissions, the power leaks during silent periods, limiting WiFi's ability to meet the minimum voltage requirements of the hardware.

power without significantly compromising network performance. This is attractive for three key reasons:

- In contrast to TV and cellular transmissions, Wi-Fi is ubiquitous in indoor environments and operates in the unlicensed ISM band where transmissions can be legally optimized for power delivery. Repurposing Wi-Fi networks for power delivery can ease the deployment of RFpowered devices without additional power infrastructure.

- Wi-Fi uses OFDM, an efficient waveform for power delivery because of its high peak-to-average ratio [38, 39]. Given Wi-Fi's economies of scale, Wi-Fi chipsets provide a cheap platform for sending these power-optimized waveforms, enabling efficient power delivery.

- Sensors and mobile devices are increasingly equipped with $2.4 \mathrm{GHz}$ antennas for communication via Wi-Fi, Bluetooth or ZigBee. We can, in principle, use the same antenna for both communication and $\mathrm{Wi}-\mathrm{Fi}$ power harvesting with a negligible footprint on the device size.

The key challenge for power delivery over $\mathrm{Wi}-\mathrm{Fi}$ is the fundamental mismatch between the requirements for power delivery and the Wi-Fi protocol. To illustrate, Fig. 1 plots the voltage at a tuned harvester in the presence of Wi-Fi transmissions. While the harvester can gather energy during WiFi transmissions, the energy leaks during silent periods. In this case, the Wi-Fi transmissions cannot meet the platform's minimum voltage requirement. Unfortunately for power delivery, silent periods are inherent to a distributed medium access protocol such as Wi-Fi, in which multiple devices share the same wireless medium. Continuous transmission from the router, while optimal for power delivery, would signifi- 


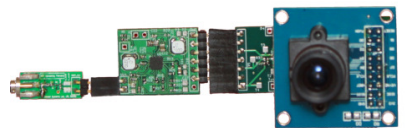

(a) Battery Free Camera

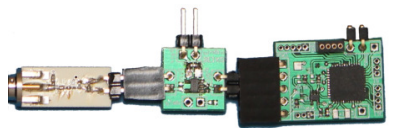

(b) Temperature Sensor

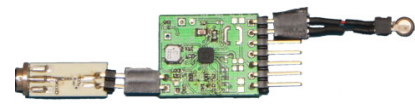

(c) Li-Ion Battery Charger

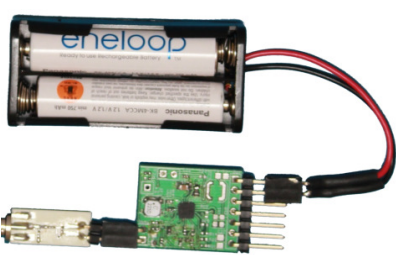

(d) NiMH Battery Charger

Figure 2-Prototype hardware demonstrating PoWiFi's potential. The prototypes harvest energy from Wi-Fi signals through a standard $2 \mathrm{dBi} \mathrm{Wi}-\mathrm{Fi}$ antenna (not shown). The low gain antenna ensures that the device is agnostic to the antenna orientation and placement. The prototypes use the harvested energy to (a) capture pictures, (b) measure temperature, and (c)/(d) recharge batteries.

cantly deteriorate the performance of Wi-Fi clients and other nearby Wi-Fi networks.

This paper introduces PoWiFi, the first power over Wi-Fi system that delivers power to energy-harvesting sensors and devices while preserving network performance. We achieve this by co-designing harvesting hardware circuits and $\mathrm{Wi}-\mathrm{Fi}$ router transmissions. At a high level, a router running PoWiFi imitates a continuous transmission while minimizing the impact on its Wi-Fi clients and other Wi-Fi networks. The key intuition is that it is unlikely that all the Wi-Fi channels are simultaneously occupied at the same instant. Thus, PoWiFi opportunistically injects superfluous broadcast traffic (which we call power packets) on non-overlapping Wi-Fi channels to maximize the cumulative occupancy across the channels. To harvest this energy, we introduce the first multichannel harvester that efficiently harvests power across multiple Wi-Fi channels and generates the 1.8-2.4 V necessary to run microcontrollers and sensor systems (see $\$ 3.2$ ).

To be practical, PoWiFi must not significantly degrade network performance. So our second component is a transmission mechanism that minimizes the impact on Wi-Fi performance while effectively providing continuous power delivery to harvesters. Specifically, to minimize the impact on associated Wi-Fi clients, PoWiFi injects power packets on a channel only when the number of data packets queued at the Wi-Fi interface is below a threshold. Further, the router transmits power packets at the highest $\mathrm{Wi}-\mathrm{Fi}$ bit rates. Since higher-rate transmissions occupy the channel for a smaller duration, PoWiFi achieves per-channel occupancies that are fair to other Wi-Fi networks.

To minimize its impact on neighboring Wi-Fi networks, PoWiFi uses two key techniques.

- Rectifier-aware transmissions. The key intuition is that when there are packets on the air, a harvester's rectifier charges exponentially, but it also discharges exponentially during silent periods. To balance power delivery and channel occupancy, PoWiFi must minimize the energy loss due to leakage. We achieve this by designing an occupancy modulation scheme that jointly optimizes the rectifier's voltage behavior and the Wi-Fi network's throughput to ensure that harvesting sensors can meet their duty-cycling requirements (see $\$ 3.1 .1$ ).

- Scalable concurrent transmissions. A key goal is to maintain good network performance when there are multiple PoWiFi routers in an area. Our insight is that PoWiFi's power packets do not contain useful data, and so the transmissions from multiple PoWiFi routers can safely collide. Further, by making each PoWiFi router transmit random power packets, we ensure that concurrent packet transmissions do not destructively interfere to reduce available power at sensors. Fig. 4 shows our transmission structure that enables multiple PoWiFi routers to co-exist.

We build PoWiFi prototypes using Atheros chipsets and build our multi-channel harvester with off-the-shelf components. Our results show the following:

- The power packets at the PoWiFi router do not noticeably affect TCP or UDP throughput or webpage load times [2] at an associated client. Meanwhile, PoWiFi achieves an average cumulative occupancy of $95.4 \%$ across the three non-overlapping $2.4 \mathrm{GHz} \mathrm{Wi}-\mathrm{Fi}$ channels.

- PoWiFi's unintrusive transmission strategy allows neighboring Wi-Fi networks to achieve better-than-equal-share fairness, because a PoWiFi router transmits power packets at the highest bit rate to minimize its channel occupancy.

- Our rectifier-aware transmission mechanism further reduces the effect on the neighboring network - it reduces the required average per-channel occupancy from $40 \%$ to $4.4 \%$, while delivering power to a sensor 16 feet away that reads temperature values once every minute.

- We perform a proof-of-concept evaluation of our concurrent transmission mechanism with 1,3 and 6 PoWiFi routers. While the variance of neighboring $\mathrm{Wi}-\mathrm{Fi}$ networks' throughput slightly increases, their mean throughput does not statistically differ. This shows the feasibility of scaling our design with multiple PoWiFi routers.

To demonstrate the potential of our design, we use our harvester to build two battery-free, Wi-Fi-powered sensing systems shown in Fig. 2: a temperature sensor and a camera. The devices use Wi-Fi power to run their sensors and a programmable microcontroller that collects the data and sends it over a UART interface. The camera and temperature-sensor prototypes can operate battery-free at distances of up to 17 and 20 feet, respectively, from a PoWiFi router. As expected, the duty cycle at which these sensors can operate decreases with distance. Further, the sensors can operate in throughthe-wall scenarios when separated from the router by various wall materials.

We also integrate our harvester with $2.4 \mathrm{~V}$ nickel-metal hydride (NiMH) and $3.0 \mathrm{~V}$ lithium-ion (Li-Ion) coin-cell 
batteries. We then build battery-recharging versions of the above sensors wherein PoWiFi trickle charges the batteries using Wi-Fi. The battery-recharging sensors can run energyneutral operations at distances of up to 28 feet.

Finally, we deploy PoWiFi routers in six homes in a metropolitan area. Each home's occupants used the PoWiFi router for their Internet access for 24 hours. Even under realworld network conditions, PoWiFi efficiently delivers power while having a minimal impact on user experience.

Contributions. We make the following contributions:

- Introduce PoWiFi, a novel system for power delivery using existing Wi-Fi chipsets. We do so without compromising the Wi-Fi network's communication performance.

- Co-design router transmissions and harvesting hardware circuits to balance power delivery and network performance. Our novel multi-channel harvester can efficiently harvest power from multiple $2.4 \mathrm{GHz} \mathrm{Wi}-\mathrm{Fi}$ channels.

- Prototype the first battery-free temperature and camera sensors that are powered using Wi-Fi chipsets. We also demonstrate the feasibility of recharging $\mathrm{NiMH}$ and LiIon coin-cell batteries using Wi-Fi signals.

- Deploy our system in six homes in a metropolitan area and demonstrate its real-world practicality.

Limitations. Given today's FCC limits in the ISM band $(1 \mathrm{~W})$, power over Wi-Fi is limited to low-power sensors and devices and can not recharge smartphones $(5 \mathrm{~W})$. Further, the range of our system is determined by the sensitivity of our harvester hardware, which is built with off-the-shelf components. We believe that an ASIC design would be able to improve the sensitivity and double PoWiFi's power-delivery range. Finally, while our current design does not account for MIMO, in principle, we can use multiple antennas to focus more power toward a sensor and increase the range, but such optimizations are beyond the scope of this paper.

\section{UNDERSTANDING WI-FI POWER}

To understand the ability of a $\mathrm{Wi}-\mathrm{Fi}$ router to deliver power, we run experiments with our organization's router and a temperature sensor. The router is an Asus RT-AC68U access point operating at $2.437 \mathrm{GHz}$ with a transmit power of $23 \mathrm{dBm}$ on each of its three $4.04 \mathrm{dBi}$ gain antennas. The temperature sensor is battery free and uses our RF harvester to draw power from Wi-Fi signals. A typical RF harvester has to provide a minimum voltage at the sensor or microcontroller to run meaningful operations. This is typically done using a rectifier that converts the carrier signal to DC and a DC-DC converter that increases the voltage level of the DC signal to match the requirements of the sensor or microcontroller. The key limitation in harvesting power is that every DC-DC converter has a minimum input voltage threshold below which it cannot operate. We use the DC-DC converter with the lowest threshold of $300 \mathrm{mV}$ [7].

We place the sensor ten feet from the router for 24 hours and measure the voltage at the rectifier output throughout our experiments. We also capture the packet transmissions from the router using a high frequency oscilloscope con- nected through a splitter. Over the tested period, the sensor could not reach the $300 \mathrm{mV}$ threshold. Fig. 1 plots both the packet transmissions and the rectifier voltage during peak network utilization. It shows that while the sensor can harvest energy during the Wi-Fi packet transmission, there is no input power during the silent slots. The hardware power leakages during these durations ensure that it does not cross the $300 \mathrm{mV}$ threshold.

\section{PoWiFi}

PoWiFi combines two elements: (1) a Wi-Fi transmission strategy that delivers power on multiple Wi-Fi channels and (2) energy-harvesting hardware that can efficiently harvest from multiple Wi-Fi channels simultaneously.

\subsection{PoWiFi Router Design}

Our key insight is that, at any moment, it is unlikely that all Wi-Fi channels will be occupied. Thus, PoWiFi opportunistically injects power packets across multiple Wi-Fi channels with a goal of maximizing cumulative occupancy. Specifically, it injects 1500-bytes UDP broadcast datagrams with a 100 us inter-packet delay at the highest $802.11 \mathrm{~g}$ bit rate of $54 \mathrm{Mbps}$ on the three non-overlapping $2.4 \mathrm{GHz} \mathrm{Wi}$ Fi channels (1, 6, and 11). A PoWiFi router enqueues these broadcast packets only when the number of frames in the wireless interface's transmit queue is below a threshold (five frames). If the queue's depth is at or above this threshold, then there are already enough power and Wi-Fi client packets in the queue to maximize channel occupancy.

PoWiFi must also provide fairness to traffic from nearby networks. Since the PoWiFi router performs carrier sensing and transmits broadcast packets at the highest $802.11 \mathrm{~g}$ bit rate, its individual frames are as short and unintrusive as possible. PoWiFi thereby provides better-than-equal-share fairness for transmissions from other Wi-Fi networks. The rest of this section describes two techniques that further reduce PoWiFi's effect on neighboring Wi-Fi networks.

\subsubsection{Rectifier-aware PoWiFi transmissions}

When PoWiFi knows a harvester's electrical characteristics, it can tune its transmission strategy to precisely fit the device's power requirements. For example, suppose we need to read a temperature sensor once per minute. PoWiFi can modulate its occupancy to deliver energy to the harvester so that the sensor reaches its required voltage of $2.4 \mathrm{~V}$ just in time, minimizing the total channel occupancy subject to this goal and thereby minimizing its effect on other networks.

Empirically modeling rectifier voltage. A rectifier converts incoming Wi-Fi transmissions into DC voltage to charge a storage capacitor. Once the voltage on the capacitor reaches the required threshold ( $V_{t h}=2.4, \mathrm{~V}$ for the temperature sensor), a reading occurs. Suppose the average power at the harvester after multi-path reflections and attenuation is $P_{\text {in }}$ and the channel occupancy of the PoWiFi router packets is $C$. To a first approximation, the harvester's behavior can be modeled as a DC voltage source charging a capacitor through a resistor. The difference, however, is that the approximated 


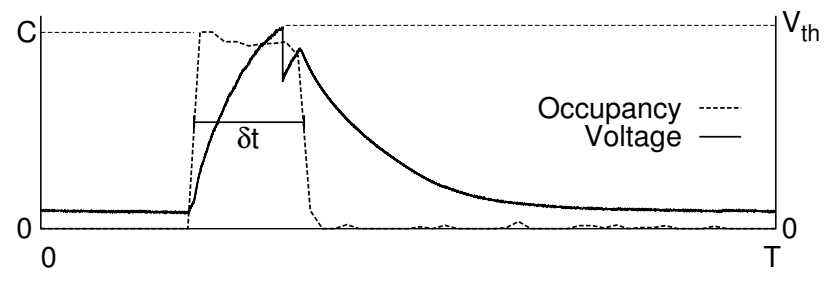

Figure 3-Rectifier-aware power Wi-Fi transmissions and corresponding rectifier voltages. The plot shows the optimized rectifier aware power Wi-Fi transmission and the corresponding voltage at a temperature sensor's storage capacitor (dotted line).

resistance value depends on the impedance of the harvester's diodes, which is a function of $P_{\text {in }}$ and $C$. We can write the voltage as a function of time as

$$
V(t)=V_{0} * e^{-t / \tau\left(P_{i n}, C\right)}+V_{\max }\left(P_{i n}, C\right) *\left(1-e^{-t / \tau\left(P_{i n}, C\right)}\right),
$$

where $V_{0}$ is the initial voltage, $\tau$ is the time constant, and $V_{\max }$ is the maximum achievable voltage. Note that both $\tau$ and $V_{\max }$ are functions of $P_{i n}$ and the channel occupancy.

Given the non-linearities of diodes, it is difficult to obtain closed-form solutions for $\tau\left(P_{i n}, C\right)$ and $V_{\max }\left(P_{i n}, C\right)$. We instead connected the harvester through a cabled setup to a WiFi source with variable input power and channel occupancy and measured the output voltage. We fitted the resulting data with the proposed exponential model to estimate how $\tau$ and $V_{\max }$ vary with input power and channel occupancy. The key properties of our model fitting are: 1) $V_{\max }$ is inverse-linearly proportional to the input power and channel occupancy; 2) the time constant $\tau$ is exponentially proportional to the input power and/or the channel occupancy; and 3) it takes exponentially more time for the same increment in the voltage at a higher voltage value than at a lower one.

We next describe how PoWiFi can modulate its channel occupancy using this empirical model, while minimizing its effect on neighboring Wi-Fi networks.

Joint optimization for efficient power delivery. To reduce the impact of power packets on neighboring Wi-Fi networks, PoWiFi must minimize the total number of power packets required to collect a sensor reading. Our key intuition is that when there are packets on the air, the capacitor charges exponentially. However, when there are no packets, the voltage on the capacitor discharges exponentially. To maximize the effectiveness of power delivery, PoWiFi must minimize capacitor leakage. We achieve this by using the channeloccupancy modulation scheme described above and shown in Fig. 3. In every sensor update time window $(T)$, the router transmits no power packets for a period $(T-\delta t)$, then transmits power packets for a period of $\delta t$, targeting a channel occupancy of $0<C \leq 1$. When the channel occupancy is zero, the voltage on the capacitor is very low and there is no leakage. However, when a sensor update is required, a high channel occupancy continuously charges the capacitor (minimizing leakage) to maximizes the effectiveness of power delivery. Our goal is to find $\delta t$ and $C$ to minimize the mean of the power packet occupancy given by $C * \frac{\delta t}{T}$.

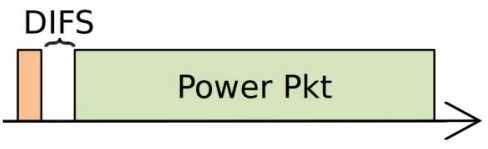

Figure 4-Energy pattern for concurrent power packet transmissions. It consists of the short packet with a 1 byte payload transmitted at $54 \mathrm{Mbps}$ followed by DIFS period and then followed by the power packet transmission.

We find these values by substituting different $C$ and $\delta t$ in our empirical model and finding the minimum value. We reduce the search space by noting that for a given $P_{i n}$, there is a minimum value of $C$ below which the threshold voltage is not achievable. Further, given a channel occupancy, we know the time constant that limits the value of $\delta t$ to a maximum value of $\tau\left(P_{i n}, C\right)$. Finally, we limit the granularity by which channel occupancy can be modulated to $10 \%$. Using these values we were able to reduce the search space to 75 points.

We note two main points. First, the above description assumes that the router can estimate the available power, $P_{i n}$, at the sensor. To bootstrap this value, PoWiFi initially transmits power packets at a high occupancy of around $90 \%$ and notes the times when the sensor outputs a reading. PoWiFi uses our empirical model to estimate $P_{i n}$ for the next cycle. At the end of every cycle it re-estimates $P_{i n}$ to account for wireless channel changes. Second, in the presence of multiple sensors, we can optimize the parameters to satisfy the minimum duty cycle requirement across all the sensors, but we omit this simple extension for brevity.

\subsubsection{Scaling with concurrent PoWiFi transmis- sions}

A practical issue with each PoWiFi router independently introducing power packets is that such a system would not preserve network performance in the presence of many PoWiFi routers. Useful Wi-Fi capacity would degrade at least linearly with the number of PoWiFi routers.

To address this scaling problem, we enable concurrent transmissions from PoWiFi routers that are in decoding range of one another. Our key insight is that since power packets do not contain useful data, transmissions from multiple PoWiFi routers can safely collide. Further, if each PoWiFi router transmits a random power packet, we can ensure that concurrent packet transmissions do not destructively interfere to reduce the power available to harvesters.

Specifically, in our system, we have a leader PoWiFi router that transmits the energy pattern shown in Fig. 4. The pattern consists of a short packet with a 1-byte payload transmitted at $54 \mathrm{Mbps}$, followed by a DIFS period and then a power packet. Other PoWiFi routers decode this short packet and join the packet transmission of the leader router within the DIFS period. This strategy ensures that all nearby PoWiFi routers transmit power packets concurrently and hence do not reduce the Wi-Fi network's capacity.

Similar to [15], we enable concurrent transmissions from the follower routers in software by setting $C W_{\min }$ and $C W_{\max }$ to 1 , preventing carrier sense backoff by setting the noise floor registers to "high" and placing their power packets in the high-priority queue. However, PoWiFi could not turn around and begin transmission within from the software 
layer within a DIFS duration. However, we believe that with better access to the router's hardware queues, PoWiFi could turn around within a DIFS period. Second, one can design distributed algorithms to find the leader router whose transmissions can be decoded by all other PoWiFi routers, but we consider this to be outside the scope of this paper.

\subsection{Multi-Channel Harvester Design}

The primary goal of our harvester design is to efficiently harvest across multiple $2.4 \mathrm{GHz}$ Wi-Fi channels. Related but equally important is to achieve good sensitivities across these channels. Sensitivity is the lowest power at which the harvester can boot up and power the sensors and the microcontroller. In theory, one can wait for a long time and harvest enough power to boot up the sensors, however, in practice, due to power leakage, a harvester cannot operate below a minimum power threshold. This is critical because the power available at the sensor decreases with distance from the Wi-Fi router; thus, the harvester's sensitivity determines its maximum operational range.

Challenge: The key challenge in the design of a Wi-Fi harvester is the impedance mismatch between the Wi-Fi antenna and the harvester. To understand this, consider a wave entering a boundary between two different mediums. If the impedance of the two mediums differ, a fraction of the incident energy is reflected. Similarly, when the antenna and the harvester have different impedance values, a fraction of the RF signal is reflected back, reducing the available RF power.

As shown in Fig. 5, a typical harvester consists of an antenna is followed by a rectifier that converts the $2.4 \mathrm{GHz}$ signal into low voltage DC power. This power is fed into a DC-DC converter that increases the voltage of the DC signal to match the voltage requirements of the sensor and microcontroller (1.8-2.4 V). The problem is that the rectifier hardware is extremely non-linear with input power, operational frequency and the parameters of the DC-DC converter, making it challenging to achieve good harvester sensitivity and efficiency across the $72 \mathrm{MHz}$ band that spans the three Wi-Fi channels.

Our Approach: As shown in Fig. 5, we design a matching network to transform the rectifier's impedance to match that of the antenna. This is, however, not straightforward because the rectifier's impedance varies significantly with frequency, power, and is dependent on the DC-DC converter. Our approach is to co-design all the components in the harvesterthe matching network, rectifier, and DC-DC converter. Our intuition is that the input of the DC-DC converter affects the input impedance of the rectifier. Thus, if we can co-design the rectifier with the DC-DC converter, we can relax the constraints on the matching network and simultaneously achieve good impedance matching across the $72 \mathrm{MHz}$ Wi-Fi band and high voltage at the output of the rectifier.

Design Details: The rest of the section describes each of the above components-rectifier, DC-DC converter, and matching network-in detail.

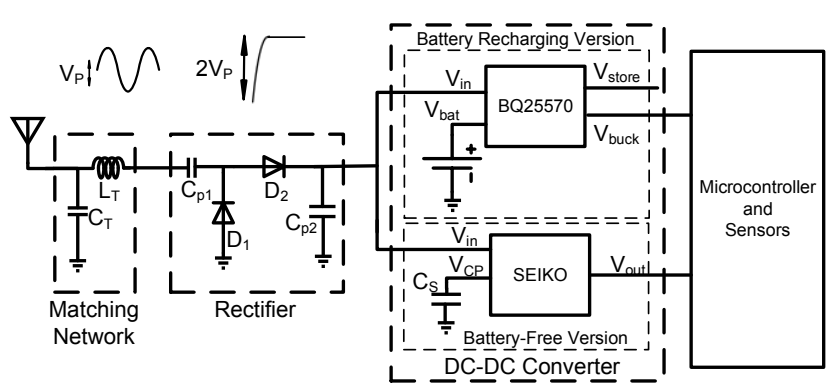

Figure 5-PoWiFi harvester schematic. PoWiFi codesigns the matching network, rectifier, and DC-DC converter to achieve good impedance matching across Wi-Fi bands. The figure shows the optimized DC-DC converters for both battery-free and battery-recharging versions of our harvester.

1) Rectifier Design. The key design consideration for rectifiers is that DC-DC converters cannot operate below a minimum input voltage. Thus, the rectifier must be designed to maximize its output voltage. Fig. 5 shows the various components used in our rectifier design. At a high level, our rectifier tracks twice the envelope of the incoming signal and converts it into power. Specifically, it adds the positive and negative cycles of the incoming sinusoidal carrier signal to double the amplitude. To do this, it uses a specific configuration of diodes and capacitors as shown in Fig. 5. However, in practice, diodes and capacitors have losses that limit the output voltage of the rectifier. We use SMS7630-061 diodes by Skyworks [8] in ultra-miniature 0201 SMT packages which low losses, i.e., loss threshold voltage, low junction capacitance and minimal package parasitics. We also use high-quality-factor, low-loss UHF-rated $10 \mathrm{pF}$ capacitors that minimize losses and maximize the rectifier's efficiency and sensitivity.

2) $D C-D C$ converter design. In our design, a DC-DC converter serves two purposes: i) boost the voltage output of the rectifier to the levels required by the microcontroller and sensors, and ii) make the input impedance of the rectifier less variable across the three Wi-Fi channels. The key challenge is the cold-start problem: in a battery-free design, all the hardware components must boot up from $0 \mathrm{~V}$. Practical DC-DC converters, however, have a nonzero minimum voltage threshold. We use the SZ882 DC-DC converter from Seiko [7], which is the best in its class: it can start from input voltages as low as $300 \mathrm{mV}$, which our rectifier can provide, and boost the output on a storage capacitor to $2.4 \mathrm{~V}$. Once the $2.4 \mathrm{~V}$ threshold is reached, the Seiko charge pump connects the storage capacitor to the output, powering the microcontroller and sensors.

A DC-DC converter can be further optimized while recharging a battery. Specifically, the battery can provide a minimum voltage level and hence the hardware components need not boot up from $0 \mathrm{~V}$. We use the TI bq25570 energyharvesting chip [3] that contains a boost converter, a battery charger, voltage monitoring solutions and a buck converter. We connect the rechargeable battery to the battery charging node, $V_{b a t}$, of the bq25570. We use the boost as our DC-DC converter to achieve the voltage required to charge the bat- 
tery. Finally, we leverage the maximum power point tracking (MPPT) mode of the TI chip to tune the input impedance of the DC-DC converter so as to minimize the variation of the rectifier's impedance across $\mathrm{Wi}-\mathrm{Fi}$ channels. Specifically, we set the buck converter's MPPT reference voltage to $200 \mathrm{mV}$.

3) Matching Network Design: With our rectifier and DCDC converter designs, we have relaxed the constraints on the impedance-matching network. The resulting circuit can match impedances between the rectifier and a $50 \Omega$ antenna across Wi-Fi channels, using a single-stage LC matching network. In LC matching networks, inductors are the primary source of losses. To mitigate this, we use highfrequency inductors in 0402 footprint which have minimal parasitics and a quality factor of 100 at $2.45 \mathrm{GHz}$ [1]. The resulting matching network consumes less board area than traditional transmission lines and distributed-element-based matching networks and can be modified to meet different system parameters without losses. We use $6.8 \mathrm{nH}$ and $1.5 \mathrm{pF}$ as the LC matching network for our battery-free harvester, and $6.8 \mathrm{nH}$ and $1.3 \mathrm{pF}$ for our battery-recharging harvester.

\section{EVALUATION}

We build rectifiers for our harvester prototypes using 2layer 20 mils Rodgers 4350 substrate printed circuit boards (PCBs). Unlike FR4, Rodgers substrate has low losses at $2.4 \mathrm{GHz}$ and does not degrade the sensitivity and efficiency of our harvester. The DC-DC converter and sensor applications however, were built on a 4-layer FR4 substrate and connected to the harvester using 10 mil headers.

We implement a PoWiFi router using three Atheros AR9580 chipsets that independently run the algorithm in $\S 3.1$ on channels 1,6 , and 11 respectively. The chipsets are connected via amplifiers to $6 \mathrm{dBi} \mathrm{Wi}-\mathrm{Fi}$ antennas separated by $6.5 \mathrm{~cm}$. Our prototype router provides Internet access to its associated clients on channel 1 via NAT and transmits at $30 \mathrm{dBm}$, which is within the FCC limit for the ISM band. All our sensor and harvester benchmark evaluations were performed in a busy office network where the average cumulative occupancy was about $90 \%$.

Our router's channel occupancy includes both the power and client packets. To measure this, we use aircrack-ng's airmon-ng tool to add a monitor interface to each of the router's active wireless interfaces. To measure the router's channel occupancy on a specific interface, we start tcpdump on the monitor interface to record the radio-tap headers for all frames and their retransmissions. We use tshark to extract frames sent by the router, recording the corresponding bitrate and frame size (in bytes). We then compute the average channel occupancy as $\sum_{i \in \text { frames }_{\text {rate }} \times \text { total_duration }_{\text {tot }}}$.

\subsection{Effect on Wi-Fi clients}

Our system is designed to provide high cumulative channel occupancies for power delivery while minimizing the effect on Wi-Fi traffic. To evaluate this, we deploy a PoWiFi router and evaluate its effect on Wi-Fi traffic. We use a Dell Inspiron 1525 laptop with an Atheros chipset as a client associated with our router on channel 1.
We compare four different schemes:

- Baseline. PoWiFi is disabled on the router, i.e., the router introduces no extra traffic on any of its interfaces.

- BlindUDP. The router transmits UDP broadcast traffic at $1 \mathrm{Mbps}$ so as to maximize its channel occupancy.

- PoWiFi. The router sends UDP broadcast traffic at $54 \mathrm{Mbps}$ and uses the queue threshold check in $\$ 3.1$.

- NoQueue. The router sends UDP broadcast traffic at $54 \mathrm{Mbps}$ but disables the queue threshold check.

We evaluate PoWiFi with various Wi-Fi traffic patterns and metrics: the throughput of UDP and TCP download traffic, the page load time (PLT) of the ten most popular websites in the United States [2], and traffic on other Wi-Fi networks in the vicinity of our benchmarking network.

(a) Effect on UDP traffic. UDP is a common transport protocol used in media applications such as video streaming. We run iperf with UDP traffic to a client seven feet from the router. The client sets its $\mathrm{Wi}-\mathrm{Fi}$ bitrate to $54 \mathrm{Mbps}$ and runs five sequential copies of iperf, three seconds apart. We repeat the experiments with target UDP data rates between 1 and $50 \mathrm{Mbps}$, and measure the achieved throughput computed over $500 \mathrm{~ms}$ intervals. All the experiments are run during a busy weekday at UW CSE, with multiple other clients and 43 other Wi-Fi networks operating at $2.4 \mathrm{GHz}$.

Fig. 6(a) plots the average UDP throughput as a function of the eleven tested UDP data rates. The figure shows that BlindUDP significantly reduces throughput. With NoQueue, the router's kernel does not prioritize the client's iperf traffic over the power traffic. This results in roughly a halving of the iperf traffic's data rate as the wireless interface is equally shared between the two flows. With PoWiFi, however, the client's iperf traffic achieves roughly the same rate as the baseline. This result demonstrates that PoWiFi effectively prioritizes client traffic above its power traffic.

For the PoWiFi experiments above, Fig. 7(a) plots the CDFs of individual channel occupancies on the three $\mathrm{Wi}-\mathrm{Fi}$ channels. The figure shows that the individual channel occupancies are around 5-50\% across the channels. The mean cumulative occupancy, on the other hand is $97.6 \%$, demonstrating that PoWiFi can efficiently deliver power even in the presence of UDP download traffic.

(b) Effect on TCP traffic. Next we run experiments with TCP traffic using iperf at the client. The router is configured to run the default Wi-Fi rate adaptation algorithm. We run experiments over a duration of three hours with a total of 30 runs. In each run, we run five sequential copies of iperf, three seconds apart, and compute the achievable throughput over $500 \mathrm{~ms}$ intervals, with all the schemes described above.

Fig. 6(b) plots CDFs of the measured throughput values across all the experiments. The plot shows that BlindUDP significantly degrades TCP throughput. As before, since $\mathrm{No}$ Queue does not prioritize the client traffic over the power packets, it roughly halves the achievable throughput. PoWiFi sometimes achieves higher throughput than the baseline. This is because of channel changes that occur during the three-hour experiment duration. The general trend how- 

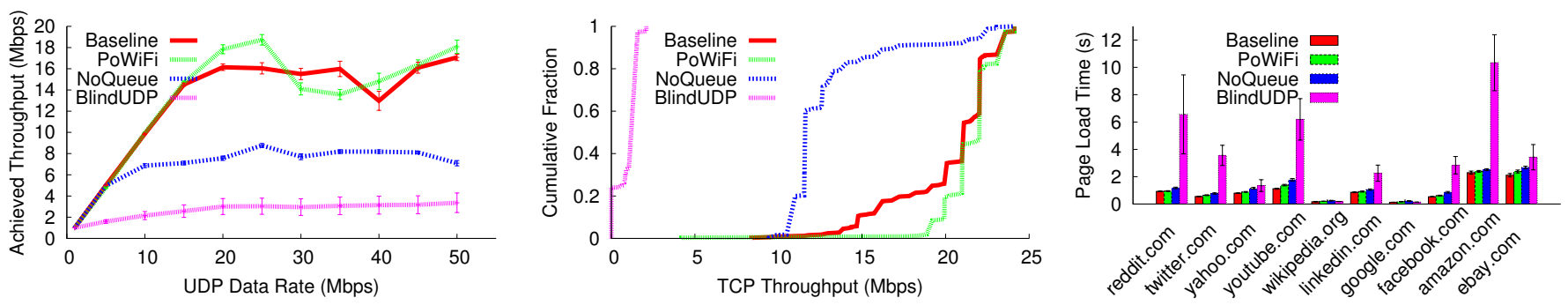

Figure 6-Effect on Wi-Fi traffic. The figures show the effect of various schemes on TCP and UDP throughput as well as the page load times of the top ten websites in the United States [2]. The plots show that PoWiFi minimizes its effect on the Wi-Fi traffic.

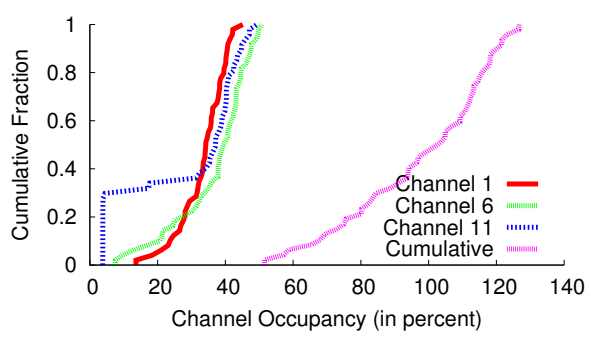

(a) UDP experiments

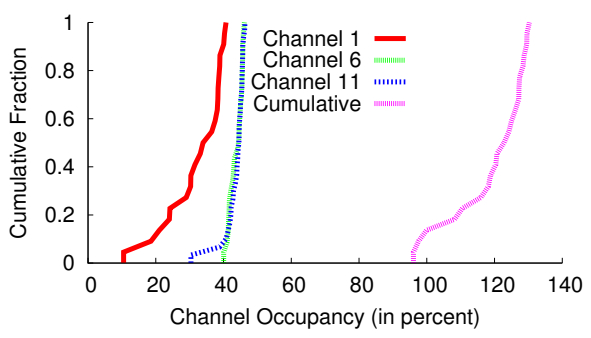

(b) TCP experiments

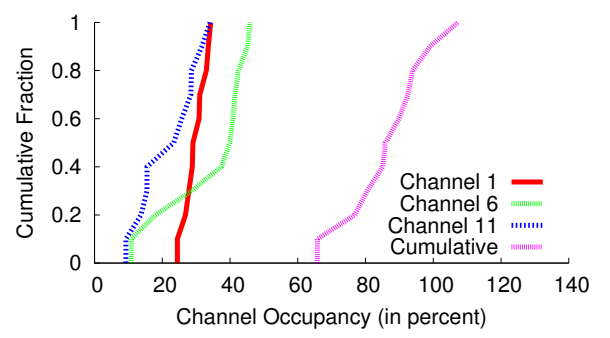

(c) PLT experiments

Figure 7-PoWiFi channel occupancies. The plots show the occupancies with PoWiFi for the above UDP, TCP, and PLT experiments.

ever points to the conclusion that PoWiFi does not have a noticeable effect on TCP throughput at the client.

Fig. 7(b) plots the CDFs of the channel occupancies for PoWiFi during the above experiments. The figure shows that PoWiFi has a mean cumulative occupancy of $100.9 \%$ and hence can efficiently deliver power.

(c) Effect on PLT. We develop a test harness that uses the PhantomJS headless browser [6] to download the front pages of the ten most popular websites in the US [2] 100 times each. We clear the cache and pause for one second in between page loads. The traffic is recorded with tcpdump and analyzed offline to determine page load time and channel occupancy. The router uses the default rate adaptation to modify its Wi-Fi bit rate. The experiments were performed during a busy weekday at UW CSE over a two-hour duration.

Fig. 6(c) shows that BlindUDP significantly deteriorates the PLT. This is expected because the $1 \mathrm{Mbps}$ power traffic occupies a much larger fraction of the medium and hence increases packet delays to Wi-Fi clients. NoQueue improves PLT over BlindUDP, with an average delay of $294 \mathrm{~ms}$ over the baseline. PoWiFi further minimizes the delay to $101 \mathrm{~ms}$, averaged across websites. This residual delay is due to the computational overhead of PoWiFi from the perpacket checks performed by the kernel. This slows down all the processes in the OS and hence results in additional delays. However, increasing processing power and moving these checks to hardware can help further reduce these delays. In our home deployments ( $\S 6)$, the users did not perceive any noticeable effects on their web performance.

For completeness, we plot the CDFs of channel occupancies for PoWiFi in Fig. 7(c). The plot shows the same trend as before, with a mean cumulative occupancy of $87.6 \%$.

\subsection{Effect on neighboring Wi-Fi networks}

(a) High cumulative channel occupancy transmissions. PoWiFi leverages the inherent fairness of the Wi-Fi MAC to ensure that it is fair to other Wi-Fi networks. As a worstcase evaluation, we consider a situation where PoWiFi always tries to achieve high cumulative channel occupancies at all times. To do this, we place our PoWiFi router in the vicinity of a neighboring Wi-Fi router-client pair operating on channel 1. We configure the PoWiFi router to transmit power aware packets at the highest achievable channel occupancies using our algorithm on all three channels. We run iperf with UDP traffic on the neighboring router-client pair at the highest data rate and measure the achievable throughput as before. We repeat the experiments for different Wi-Fi bit rates at the neighboring Wi-Fi router-client pair. We compare three schemes: BlindUDP where our router transmits UDP packets at $1 \mathrm{Mbps}$, EqualShare where we set our router to transmit the UDP packets at the same Wi-Fi bit rate as the neighboring router-client pair, and finally PoWiFi. EqualShare provides a baseline when every router in the network gets an equal share of the wireless medium.

Figure 8(a) shows the throughput for the three schemes, averaged across five runs. As expected, BlindUDP significantly deteriorates the neighboring router-client performance. Further, this deterioration is more pronounced at the higher Wi-Fi bit rates. With PoWiFi, however, the throughput achieved at the neighboring router-client pair is higher than EqualShare. This is because PoWiFi transmits power packets at $54 \mathrm{Mbps}$; transmissions at such high Wi-Fi bit rates occupy the channel for a smaller duration than, say, a neighboring router transmitting at $16 \mathrm{Mbps}$. This property means that PoWiFi provides better than equal-share fairness 
to other Wi-Fi networks. We note that while our experiments are with $802.11 \mathrm{~g}$, PoWiFi's power packets use the highest bit rate available for Wi-Fi. Thus, the above fairness property would hold true even with $802.11 \mathrm{n} / \mathrm{ac}$.

(b) Rectifier-aware power transmissions. Next we evaluate the potential of our rectifier-aware technique, to significantly reduce the average channel occupancy of the power transmissions, while efficiently delivering power to the sensors. To do this, we place our battery-free temperature sensor close to its maximum operational range at 16 feet from a PoWiFi router; the sensor is set to transmit a temperature value over a UART interface once every minute. The router implements the joint-optimization algorithm from §3.1.1.

We ran the experiments for a total of ten minutes and observed that the temperature sensor achieves a mean update rate of $59.93 \mathrm{~s}$ with a $0.43 \mathrm{~s}$ variance. More importantly, in contrast to transmitting at high channel occupancies ( $>$ 90\%) all the time, our algorithm estimated that the router should transmit for a duration of $9 \mathrm{~s}$ with a $80 \%$ cumulative occupancy and stay quiet for the remaining time. Fig. 8(b) shows the throughput of an ongoing TCP flow in a neighboring Wi-Fi router-client pair, which shows that the average throughput significantly improves over high-occupancy Po$\mathrm{WiFi}$ and is much closer to the baseline throughput without any power packets. Fig. 8(c) shows that rectifier aware transmissions have an average per-channel occupancy of 3.3\%, compared to $40 \%$ per-channel occupancy for PoWiFi transmissions - a 10x reduction in average occupancy.

(c) Scalable concurrent power transmissions. Finally, we provide a proof-of-concept evaluation of our concurrent transmission mechanism. Wi-Fi hardware is designed to turn around between decoding a packet and transmitting within a SIFS duration and hence can in principle, easily achieve the timing requirement in Fig. 4(d). Since we currently only have software access to the router, we are limited to implementing using high-speed timers and high-priority queue. Our current software system has $36.15 \mu$ s mean turn around time with $4.61 \mu$ s variance.

Using the above mean turn around time as the silence period, we do a proof-of-concept evaluation. To simplify implementation, we setup a USRP N210 to transmit the pattern in Fig. 4 at 30\% channel occupancy. The PoWiFi routers join this USRP transmission and concurrently transmit power packets. We evaluate the impact on the TCP throughput of a neighboring Wi-Fi router-client pair as we increase the number of PoWiFi routers. Fig. 8(d) shows that as the number of devices increases, the throughput variance slightly increases. This is because as the number of devices increases, the variance in the turn-around time between Wi-Fi power transmissions increases. The figure however shows that, the mean throughput is statistically unaffected as the number of PoWiFi devices increases from 1 to 6 . This shows the feasibility of scaling with multiple PoWiFi routers.

\subsection{Evaluating the Harvesting Hardware}

The harvester's performance is determined by: 1) impedance matching at the antenna interface to maximize

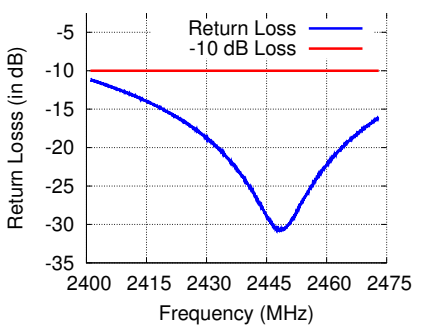

(a) Battery-free harvester

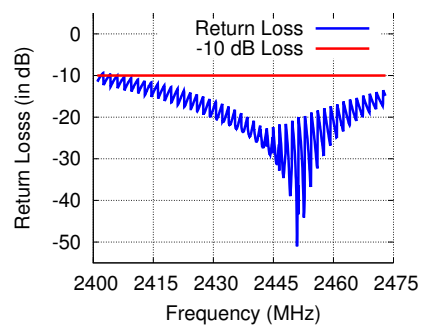

(b) Battery-charging harvester
Figure 9-Harvester return loss. This is the ratio of reflected power to the incident power. Across the $2.4 \mathrm{GHz}$ WiFi band, the return loss is less than $-10 \mathrm{~dB}$. This translates to less than $0.5 \mathrm{~dB}$ of lost power, which is negligible.

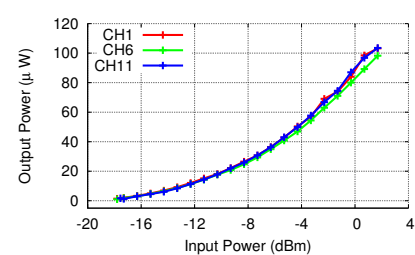

(a) Battery-free harvester

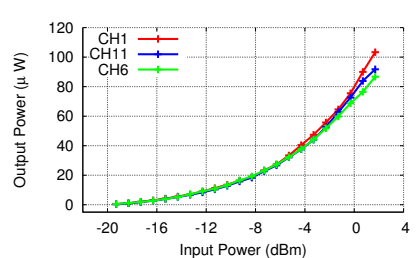

(b) Battery-charging harvester
Figure 10-Available output power at the harvester. The battery charging harvester operates at $-19.3 \mathrm{dBm}$ compared to $-17.8 \mathrm{dBm}$ for the battery free harvester which results in a higher operating range for the battery charging harvester.

the RF energy delivered to the rectifier, and 2) the rectifier's ability to convert RF energy into useful DC power.

(a) Impedance matching versus frequency. If the antenna's impedance differs from the harvester's, a portion of the incident RF signal will be reflected back and cannot be converted into DC power. The amount of reflection is determined by the impedance difference, which our matching network aims to minimize across all three Wi-Fi channels. Impedance matching performance is measured using return loss: ratio of reflected power to the incident power.

We compute the return loss by connecting the harvester to a vector network analyzer that transmits RF signals across the entire Wi-Fi band. We analyze the power reflected at each frequency to compute the return loss. Fig. 9 plots the return loss of the battery-free and battery-charging versions of our harvester. Across $2.401-2.473 \mathrm{GHz}$, both of our harvesters achieve a return loss of less than $-10 \mathrm{~dB}$, which in most RF circuits and systems is acceptable [32]. This translates to less than $0.5 \mathrm{~dB}$ of lost power, which is negligible.

(b) Available power at the rectifier output. The rectifier converts the RF signals at the harvester into DC output voltage. This conversion is typically low due to the inherent nonlinearities and threshold voltage drop of diodes. To measure the available power, we use a cable to connect our hardware to the output of a Wi-Fi transmitter and a continuous wave transmitter. We found that compared to continuous wave, Wi-Fi transmissions have $0.5 \mathrm{~dB}$ higher sensitivity which increases the operating range by $6 \%$. Next we vary the output power and the operational frequency of the Wi-Fi transmitter and measure the power available at the rectifier's output.

Fig. 10 shows the output power at the rectifier as a function of input RF power. The results are plotted for both our 


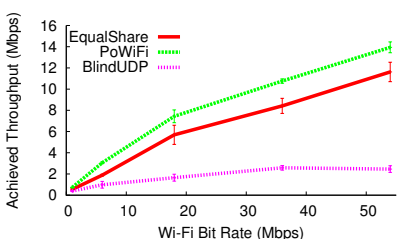

(a) PoWiFi bit-rates

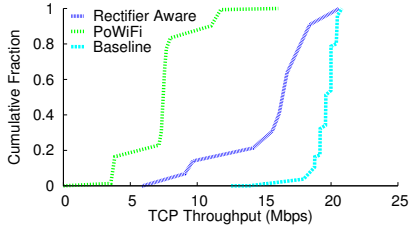

(b) Rectifier aware througput

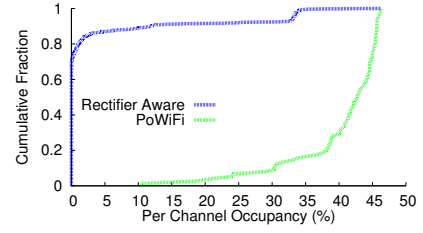

(c) Rectifier aware occupancies

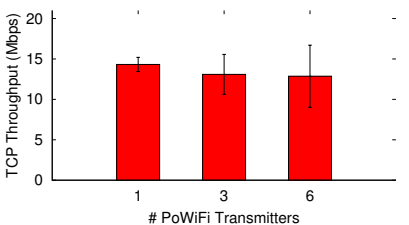

(d) Concurrent transmissions

Figure 8-Effect of PoWiFi, rectifier aware and concurrent power transmissions on neighboring Wi-Fi networks. The plots show that PoWiFi power transmissions provide better than EqualShare throughput performance. Rectifier aware power transmissions further improve the throughput by reducing the per channel occupancy by a factor of 10. Additionally, increasing the number of concurrently transmitting PoWiFi devices does not degrade the performance of neighboring Wi-Fi devices.

battery-free as well as battery-charging harvesters, across the three Wi-Fi channels. The plots show the following:

- The harvester's output power scales with the input power. For instance at a distance of 2 feet the battery charging system has $100 \mu \mathrm{W}$ available compared to $10 \mu \mathrm{W}$ at 10 feet. This means that as a harvesting sensor moves farther to the router, it can operate at a lower duty cycle.

- The battery-charging harvester operates down to $19.3 \mathrm{dBm}$, compared to $-17.8 \mathrm{dBm}$ for the battery-free harvester. This is because the battery-charging harvester does not have the cold start limitation. Specifically, a battery-free harvester has to start all its hardware components from cold start $(0 \mathrm{~V})$. In contrast, a battery-charging harvester can use the connected battery to provide a nonzero voltage value, allowing for greater sensitivities.

- Our harvesters perform efficiently across Wi-Fi channels 1,6 and 11. This is a result of our optimized multi-channel harvester design that ensures efficient power harvesting.

\section{SENSOR APPLICATIONS}

We integrate our harvesters with sensors at two ends of the energy-consumption spectrum: a temperature sensor and a camera. We build both battery-free and battery-recharging versions of each sensor.

\subsection{Wi-Fi powered Temperature Sensor}

We use our harvester to power an LMT84 temperature sensor and an MSP430FR5969 microcontroller to read and transmit sensor data. We optimize our sensor for power and the entire measurement and data-transmission operation uses only $2.77 \mu \mathrm{J}$. The battery-recharging sensor, on the other hand, consists of our rectifier followed by the TI bq25570 power-management chip to wirelessly recharge two AAA $750 \mathrm{mAh}$ low discharge current NiMH battery at $2.4 \mathrm{~V}$ (see our tech report [35] for more details).

Experiments. We evaluate the effect of distance on the update rate of the temperature sensor. Specifically, we use a PoWiFi router and place both the battery-recharging and battery-free sensor at increasing distances. In the batteryfree case, we measure the update rate by computing the time between successive sensor readings. In the battery-operated case, we measure the battery voltage and the charge current flowing into it from the harvester. Since, each temperature measurement and data transmission takes $2.77 \mu \mathrm{J}$, we compute the ratio of the incoming power to this value to ascertain

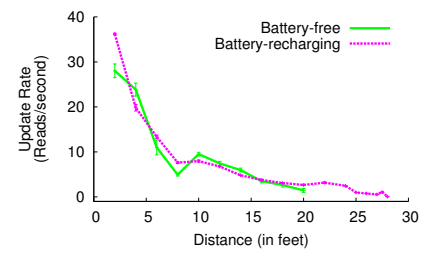

(a) Temperature Sensor

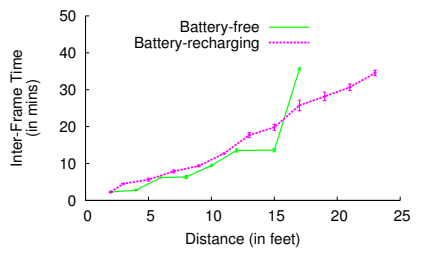

(b) Camera
Figure 11-Sensor update rate. The temperature (camera) sensor can operate up to 20 (17) and 28 (23) feet as batteryfree and energy-neutral battery-recharging respectively. This enables low-rate cameras to be left in hard-to-reach places such as walls, attics, and sewers for leakage and structural integrity detection, without the need for replacing batteries.
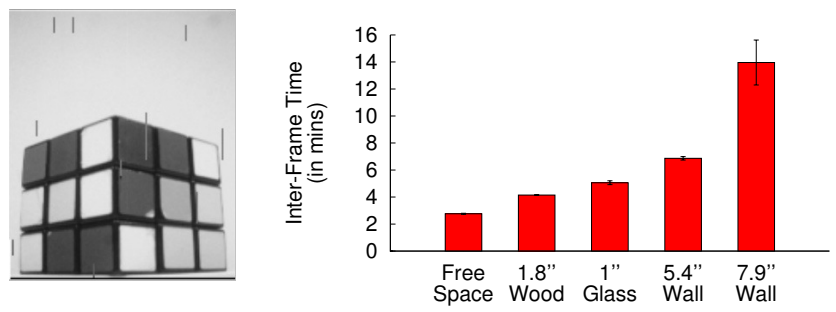

Figure 12-Battery-free camera in through-the-wall scenarios. The figure on the left is a picture of a Rubik's cube taken with our camera prototype. The plot shows the interframe time with different wall materials at a five feet distance from the router.

the sensor update rate for energy-neutral operation. The average cumulative occupancy in our experiments was $91.3 \%$.

Results. Fig. 11 plots the results for both our sensors. The update rates decrease with the distance from the router. This is a result of less power being harvested and agrees with the harvester benchmarks in $\$ 4.3$. At closer distances, both harvesters have similar update rates. Beyond 15 feet, however, the battery-powered sensor, optimized for lower input power, has a better update rate and extended operational range: it can operate up to 20 feet from the router. The battery-recharging sensor can operate in an energy-neutral manner to greater distances of up to 28 feet.

\subsection{Wi-Fi powered Camera}

We use OV7670, a low-power VGA image sensor from Omnivision, and interface it with an MSP430FR5969 microcontroller. We optimize our firmware for power and achieve a per-image capture energy of $10.4 \mathrm{~mJ}$. On our batteryfree camera, we use an ultra-low leakage AVX BestCap $6.8 \mathrm{mF}$ super-capacitor as the storage element. Our battery- 
recharging camera consists of the same hardware as before, but uses our wirelessly rechargeable $1 \mathrm{mAh}$ lithium-ion coin-cell battery at 3.0 V (see [35] for details).

Experiments 1. We evaluate the time between frames as a function of distance for both our prototypes. As before, we use a PoWiFi router-the observed average cumulative occupancy was $90.9 \%$ across experiments. At each distance from the router, we wait for the camera to take at least six frames and measure the time interval between consecutive frames. For the battery-recharging camera, we ascertain the inter-frame duration for an energy-neutral image capture.

Results 1. Fig. 11(b) shows that the battery-free camera can operate up to 17 feet from the router, with an image capture every 35 minutes. On the other hand, the battery-recharging camera has an extended range of 23 feet with an image capture every 34.5 minutes in an energy-neutral manner. Both the sensors have a similar image capture rate up to 15 feet from the router. We also note that Fig. 11(b) limits the range to 23 feet to focus on the smaller values. Our experiments, however, show that the battery-recharging camera can operate up to 26.5 feet with an image capture every 2.6 hours.

A key question the reader should ask is: would cameras with such low image-capture rate be useful in practice? Taking a picture periodically, as above, is an artificial construct of our experiment. In practice, we could integrate our camera with motion-detection sensors that consume orders of magnitude lower power [23] and turn on the camera only when motion is detected. Another application is to use these cameras in hard-to-reach places such as walls, attics, pipes and sewers for leakage and structural-integrity detection. In these scenarios, replacing batteries can be cumbersome, and our low rate camera sensor would be an effective solution.

Experiments 2. Motivated by the above applications, we next evaluate our camera in through-the-wall scenarios. We place our PoWiFi router next to a wall and place our batteryfree camera prototype 5 feet away on the other side of the wall. We experiment with walls of four different materials: a double-pane glass wall one inch thick, a wooden door 1.8 inches thick, a hollow wall 5.4 inches thick, and finally a double sheet-rock (plus insulation) wall with a thickness of 7.9 inches.

Results 2. Fig. 12 shows the mean time between frames, averaged over five frames, as a function of the material. The plot shows that as the material absorbs more signals (e.g., double sheet-rock versus glass), the time between frames increases. However, the key conclusion is that PoWiFi can power cameras through walls and enable applications where the cameras can be left in hard-to-reach places such as walls and sewers, without the need for replacing batteries.

\section{HOME DEPLOYMENT STUDY}

In $\$ 4.2$ we showed that the channel occupancy of PoWiFi can be optimized for different sensor applications and minimize impact on neighboring Wi-Fi devices. However, PoWiFi's ability to efficiently deliver power depends on the traffic patterns of other Wi-Fi networks in the vicinity as
Table 1 -Summary of our home deployment

\begin{tabular}{|c|cccccc|}
\hline Home \# & $\mathbf{1}$ & $\mathbf{2}$ & $\mathbf{3}$ & $\mathbf{4}$ & $\mathbf{5}$ & $\mathbf{6}$ \\
\hline Users & 2 & 1 & 3 & 2 & 1 & 3 \\
\hline Devices & 6 & 1 & 6 & 4 & 2 & 6 \\
\hline Neighboring APs & 17 & 4 & 10 & 15 & 24 & 16 \\
\hline
\end{tabular}

well as the router's own client traffic, both of which can be unpredictable. So we deploy our system in six homes in a metropolitan area and measure PoWiFi's ability to continuously achieve high channel occupancies.

Table 1 summarizes the number of users, devices and other $2.4 \mathrm{GHz}$ routers nearby in each of our deployments. We replace the router in each home with a PoWiFi router, and the occupants use it for normal Internet access for 24 hours. Our router uses the same SSID and authentication information as the original router, which we disconnect. We place our router within a few feet of the original router, with the exact location determined by user preferences. In all six deployments, we set our router to provide Internet connectivity on channel 1 and to transmit power packets on channels 1,6 , and 11 using the algorithm in $\$ 3.1$. We stage our deployment over the period of a week-first two homes in Table 1 over a weekend and the rest on weekdays.

We log the router's channel occupancy on each of the three Wi-Fi channels at a resolution of $60 \mathrm{~s}$. Fig. 13 plots the occupancy values for each Wi-Fi channel over the 24hour deployment duration. We also plot the cumulative occupancy across the channels. The figures show that:

- We see significant variation in per-channel occupancy across homes. This is because when the load is high on neighboring networks, our router scales back its transmissions on that channel and has lower channel occupancy. However, when the load on neighboring networks is low, the router occupies a larger fraction of the wireless channel. This is because PoWiFi uses carrier sense to enforce fairness with other Wi-Fi networks.

- The cumulative occupancy is high over time in all our home deployments. Specifically, the mean cumulative occupancies for the six home deployments are in the 78$127 \%$ range. We note that some of these occupancies are much greater than $100 \%$, which might not be necessary for power delivery. One can however reduce the perchannel rate of the power traffic based on the cumulative occupancy value to ensure that it is below $100 \%$. Our current system does not implement this feature.

- The users in homes 1-4 did not perceive any noticeable difference in their user experience. The user in home 5, however, noted a significant improvement in page load times and better experience on streaming sites including Hulu, Amazon Prime and YouTube. This was primarily because home 5 originally was using a cheap low-grade router with worse specifications. A user in home 6 noted a slight deterioration in YouTube viewing experience for a 30-minute duration. Our analysis showed that our router occupancy, including both client and power traffic, dipped 


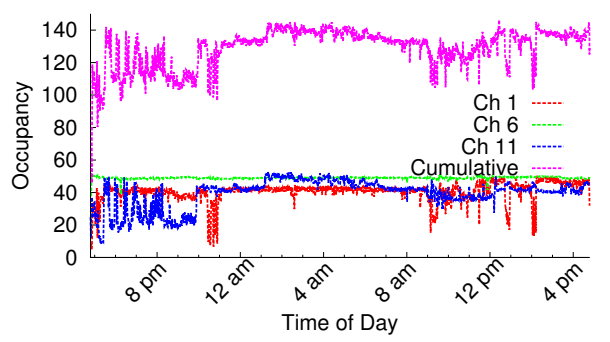

(a) Home 1

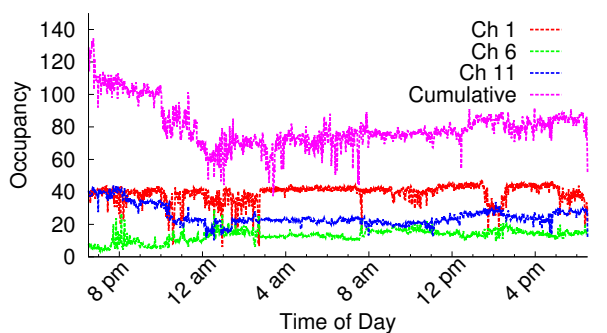

(d) Home 4

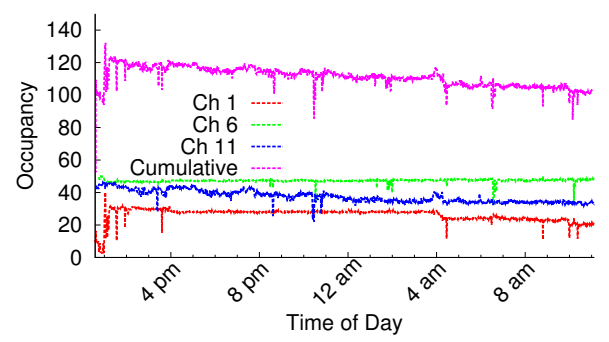

(b) Home 2

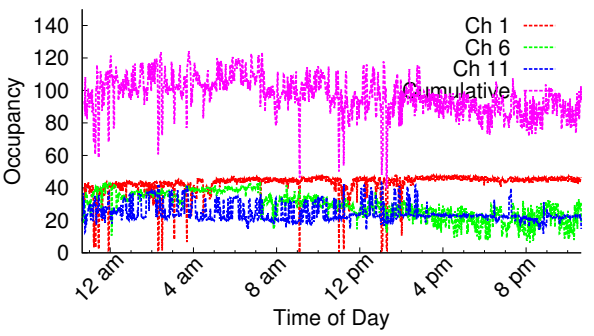

(e) Home 5

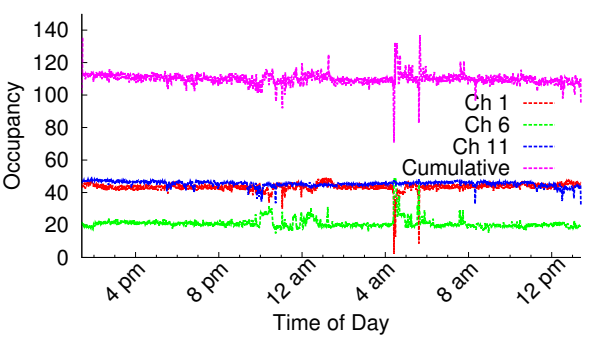

(c) Home 3

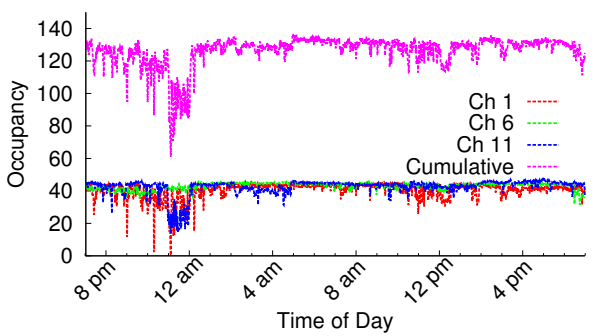

(f) Home 6

Figure 13-PoWiFi channel occupancies in home deployments. We see significant variation in per-channel occupancy values across homes. This is because PoWiFi uses carrier sense that reduces its occupancy when the neighboring networks are loaded. The cumulative occupancy, however, is high across time in all home deployments. We note that, in principle, one can modify PoWiFi's algorithm to reduce the per-channel occupancy of the power traffic and keep the cumulative occupancy less than $100 \%$, which is sufficient for harvesting purposes.

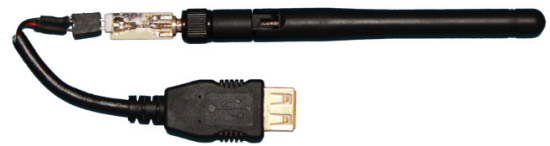

Figure 14-Wi-Fi power via USB. It consists of a $2 \mathrm{dBi}$ Wi-Fi antenna attached to our harvester. Using this, we charge a Jawbone UP24 device in the vicinity of the Po$\mathrm{WiFi}$ router from a no-charge state to $41 \%$ charged state in 2.5 hours.

during this duration. This points to external causes including interference from other devices in the environment.

\section{ROUTER AS A CHARGING HOTSPOT}

In addition to powering custom temperature and camera sensors, PoWiFi can transform the vicinity of a Wi-Fi router into a wireless charging hotspot for devices such as wearable activity trackers. To demonstrate the feasibility of this, we design the general-purpose USB charger shown in Fig. 14. It consists of a $2 \mathrm{dBi} \mathrm{Wi}-\mathrm{Fi}$ antenna attached to a custom harvester that we optimize for higher input power. We then connect our USB charger to a Jawbone UP24 device and place it 5-7 cm away from the PoWiFi router. We observe that the charger could supply an average current of $2.3 \mathrm{~mA}$ and charge the Jawbone UP24 battery from a no-charge state to $41 \%$ charged-state in 2.5 hours. This demonstrates the potential of our approach. We are currently working on designs that would directly integrate our harvester with the antenna of the wearable device. Further, we are exploring the use of a custom battery charging solution, similar to those demonstrated in this paper, to achieve higher efficiencies and longer-distance wireless charging for these devices.

\section{RELATED WORK}

Wireless power delivery techniques can be primarily divided into two categories: near-field magnetic resonance/inductive coupling $[11,24]$ and RF power transmission systems. Of the two, RF power delivery is the truly long-range mechanism and hence we focus on the latter category.

Early RF power delivery systems were developed as part of RFID systems to harvest small amounts of power from a dedicated $900 \mathrm{MHz}$ UHF RFID readers [34]. The power harvested from RFID signals has been used to operate accelerometers [34], temperature sensors [34], and recently cameras [27]. Our efforts on power delivery over Wi-Fi are complimentary to RFID systems. In principle, one can combine multiple ISM bands including $900 \mathrm{MHz}, 2.4 \mathrm{GHz}$, and $5 \mathrm{GHz}$ to design an optimal power delivery system. This paper takes a significant step towards this goal.

Recently, researchers have demonstrated the feasibility of harvesting small amounts of power from ambient TV [21,25] and cellular base station signals $[31,41]$ in the environment. While TV and cellular signals are stronger in outdoor environments, they are significantly attenuated indoors limiting the corresponding harvesting opportunities. The ability to power devices using Wi-Fi can augment the above capabilities and enable power harvesting indoors.

Researchers have explored the feasibility of harvesting power in the $2.4 \mathrm{GHz}$ ISM band $[10,13,14,17,18,20,28-$ $30,36,40]$. These efforts have demonstrated power harvesting from continuous wave $(\mathrm{CW})$ transmissions ${ }^{1}$ and none have powered devices with existing Wi-Fi chipsets. Fur-

\footnotetext{
${ }^{1}$ Continuous wave transmissions are special signals that have a constant amplitude and a single frequency tone.
} 
Table 2-Comparison of our harvester with the state of the art

\begin{tabular}{|c|c|c|c|c|c|}
\hline \multicolumn{7}{|c}{ RF Source } & \multicolumn{2}{c|}{ Sensitivity } & Bandwidth & Startup & Application Evaluation \\
\hline PoWiFi & Wi-Fi & $-17.8 \mathrm{dBm} @ 2.4 \mathrm{~V}$ & $100 \mathrm{MHz}$ & Cold start & Temperature/camera \\
\hline PoWiFi & Wi-Fi & $-19.3 \mathrm{dBm} @ 2.4 / 3.0 \mathrm{~V}$ & $100 \mathrm{MHz}$ & Self start & Recharge battery \\
\hline$[16]$ & $\mathrm{CW}$ & $-25 \mathrm{dBm} @ 2.4 \mathrm{~V}$ & N/A & Push button & Recharge battery \\
\hline$[10]$ & $\mathrm{CW}$ & $-20 \mathrm{dBm} @ 100 \mathrm{mV}$ & $75 \mathrm{MHz}$ & Cold start & Rectifier loaded by $8.2 \mathrm{k} \Omega$ \\
\hline$[28]$ & $\mathrm{CW}$ & $-20 \mathrm{dBm} @ 125 \mathrm{mV}$ & $100 \mathrm{MHz}$ & Cold start & Rectifier loaded by $10 \mathrm{k} \Omega$ \\
\hline$[20]$ & Microwave oven & $-10 \mathrm{dBm} @ 150 \mathrm{mV}$ & N/A & Cold start & Rectifier loaded by $10 \mathrm{k} \Omega$ \\
\hline
\end{tabular}

ther, $[17,18,20]$ harvest from incoming signals in excess of $-5 \mathrm{dBm}$ and can operate only in close proximity of the power source. $[10,29]$ design a rectifier that outputs voltages around $100 \mathrm{mV}$ for continuous wave transmissions at specific frequency tones. It is unclear how one may transform this into $1.8-2.4 \mathrm{~V}$ required by microcontrollers, sensors and batteries. [13] discusses an IC implementation of a $2.45 \mathrm{GHz}$ continuous-wave RFID tag. [14] has recently analyzed the impact of the bursty nature of Wi-Fi traffic on the rectifier and optimizes the size of the rectifier's output capacitor based on Wi-Fi burstiness. However, similar to [10,29], this work is focused on rectifier design and does not power sensors and microcontrollers or recharge batteries. We also note that our work takes a different approach to the problem: we mask the burstiness in Wi-Fi traffic and instead create high cumulative channel occupancy at the router. [16] designs an efficient $2.4 \mathrm{GHz}$ rectenna patch and battery charging solution which requires a mechanical push button for startup. The system is evaluated with continuous wave transmissions in an anechoic chamber, and not Wi-Fi signals. In contrast, PoWiFi is the first power over Wi-Fi system that works with existing Wi-Fi chipsets and minimizes its impact on Wi-Fi performance. Table 2 shows a summary comparison of our harvester with the state of the art $2.4 \mathrm{GHz}$ harvesters.

Our work is also related to efforts from startups such as Ossia [4] and Wattup [9]. These efforts claim to deliver around $1 \mathrm{~W}$ of power at ranges of 15 feet and charge a mobile phone [5]. Back-of-the-envelope calculations however show that this requires continuous transmissions with an EIRP (equivalent isotropic radiated power) of $83.3 \mathrm{dBm}$ $(213 \mathrm{~kW})$. This not only jams the Wi-Fi channel but also is 50,000 times higher power than that allowed by FCC regulations part 15 for point to multi-point links. In contrast, our system is designed to operate within the FCC limits and has minimal impact on Wi-Fi traffic. We note that in the event of an FCC exception to these startups, our multi-channel design can be used to deliver such high power without having significant impact on Wi-Fi performance.

Finally, recent work on Wi-Fi backscatter [22] enables low-power connectivity with existing Wi-Fi devices. Backscatter communication is orders of magnitude more power-efficient than traditional radio communication and hence enables Wi-Fi connectivity without incurring Wi-Fi's power consumption. However, [22] is focused on the communication mechanism and to the best of our knowledge, does not evaluate the feasibility of delivering power using Wi-Fi. Our work is complementary to [22] and can in prin- ciple be combined to achieve both power delivery and lowpower connectivity using Wi-Fi devices.

\section{CONCLUSION}

There is increasing interest in the Internet-of-Things where small computing sensors and mobile devices are embedded in everyday objects and environments. A key issue is how to power these devices as they become smaller and more numerous; plugging them in to provide power is inconvenient and is difficult at large scale. We introduce a novel far-field power delivery system using existing Wi-Fi chipsets. We do so while minimizing the impact on Wi-Fi network performance. While this is a first step towards using Wi-Fi chipsets for power delivery, we believe that with subsequent iterations of the harvester design we can significantly increase the capabilities of our system.

Acknowledgements: This research is funded in part by NSF grants CNS-1452494 and CNS-1407583, a Qualcomm Innovation Fellowship, a Intel Fellowship and University of Washington. Finally, we thank the anonymous CoNEXT reviewers for their helpful comments.

\section{REFERENCES}

[1] $0402 \mathrm{HP}$ Series Inductors by Coilcraft. http://www.coilcraft.com/pdfs/0402hp.pdf.

[2] Alexa - Top Sites in United States. http://www.alexa.com/topsites/countries/US. Loaded January 13, 2015.

[3] bq25570 by Texas Instruments. http://www.ti.com/lit/ds/symlink/bq25570.pdf.

[4] Cota by Ossia. http://www.ossiainc.com/.

[5] Energous Wattup wireless charging demo. http://www.engadget.com/2015/01/05/ energous-wattup-wireless-charging-demo/.

[6] PhantomJS. http://phantomjs.org/. Loaded January 14, 2015.

[7] S-882Z Series by SEIKO. http://www.eet-china.com/ ARTICLES/2006MAY/PDF/S882Z_E.pdf.

[8] SMS7630-061 by Skyworks. http://www.skyworksinc.com/uploads/documents/ SMS7630_061_201295G.pdf.

[9] Wattup by Energous. http://www.energous.com/overview/.

[10] G. Andia Vera, A. Georgiadis, A. Collado, and S. Via. Design of a $2.45 \mathrm{ghz}$ rectenna for electromagnetic (em) energy scavenging. In IEEE RWS 2010.

[11] W. P. Consortium. Qi wireless power specification.

[12] G. Covic and J. Boys. Inductive power transfer. Proceedings of the IEEE, 2013.

[13] J.-P. Curty, N. Joehl, C. Dehollaini, and M. J. Declercq. Remotely powered addressable uhf rfid 
integrated system. IEEE Journal of Solid-State Circuits, 2005

[14] J. Ensworth, S. Thomas, S. Y. Shin, and M. Reynolds. Waveform-aware ambient rf energy harvesting. In IEEE RFID 2014.

[15] S. Gollakota, N. Ahmed, N. Zeldovich, and D. Katabi. Secure in-band wireless pairing. In USENIX Security, 2011.

[16] K. Gudan, S. Shao, J. J. Hull, J. Ensworth, and M. S. Reynolds. Ultra-low power $2.4 \mathrm{ghz}$ rf energy harvesting and storage system with $-25 \mathrm{dbm}$ sensitivity. In IEEE RFID 2015.

[17] J. A. Hagerty, F. B. Helmbrecht, W. H. McCalpin, R. Zane, and Z. B. Popovic. Recycling ambient microwave energy with broad-band rectenna arrays. IEEE Transactions on Microwave Theory and Techniques, 2004.

[18] A. M. Hawkes, A. R. Katko, and S. A. Cummer. A microwave metamaterial with integrated power harvesting functionality. Applied Physics Letters, 2013.

[19] J. Jadidian and D. Katabi. Magnetic mimo: How to charge your phone in your pocket. MOBICOM, 2014.

[20] Y. Kawahara, X. Bian, R. Shigeta, R. Vyas, M. M. Tentzeris, and T. Asami. Power harvesting from microwave oven electromagnetic leakage. In UbiComp 2013.

[21] Y. Kawahara, H. Lee, and M. M. Tentzeris. Sensprout: Inkjet-printed soil moisture and leaf wetness sensor. In Ubicomp 2012.

[22] B. Kellogg, A. Parks, S. Gollakota, J. Smith, and D. Wetherall. Internet connectivity for rf-powered devices. SIGCOMM, 2014.

[23] B. Kellogg, V. Talla, and S. Gollakota. Bringing gesture recognition to all devices. In Usenix NSDI, 2014.

[24] A. Kurs, A. Karalis, R. Moffatt, J. D. Joannopoulos, P. Fisher, and M. Soljacic. Wireless power transfer via strongly coupled magnetic resonances. Science, 2006.

[25] V. Liu, A. Parks, V. Talla, S. Gollakota, D. Wetherall, and J. R. Smith. Ambient Backscatter: Wireless Communication Out of Thin Air. In ACM SIGCOMM, 2013

[26] Z. N. Low, R. Chinga, R. Tseng, and J. Lin. Design and test of a high-power high-efficiency loosely coupled planar wireless power transfer system. Industrial Electronics, IEEE Transactions on, 2009.

[27] S. Naderiparizi, A. Parks, Z. Kapetanovic, B. Ransford, and J. R. Smith. Wispcam: A battery-free rfid camera. In IEEE RFID 2015.
[28] U. Olgun, C.-C. Chen, and J. Volakis. Efficient ambient wifi energy harvesting technology and its applications. In IEEE APSURSI 2012.

[29] U. Olgun, C.-C. Chen, and J. Volakis. Wireless power harvesting with planar rectennas for $2.45 \mathrm{ghz}$ rfids. In URSI 2010.

[30] U. Olgun, C.-C. Chen, and J. Volakis. Design of an efficient ambient wifi energy harvesting system. IET Microwaves, Antennas Propagation, 2012.

[31] A. N. Parks, A. P. Sample, Y. Zhao, and J. R. Smith. A wireless sensing platform utilizing ambient rf energy. In IEEE BioWireless 2013

[32] D. M. Pozar. Microwave engineering. John Wiley \& Sons, 2009.

[33] A. Sample and J. R. Smith. Experimental results with two wireless power transfer systems. In IEEE RWS 2009.

[34] A. Sample, D. Yeager, P. Powledge, A. Mamishev, and J. Smith. Design of an rfid-based battery-free programmable sensing platform. IEEE Transactions on Instrumentation and Measurement, 2008.

[35] V. Talla, B. Kellogg, B. Ransford, S. Naderiparizi, S. Gollakota, and J. R. Smith. Powering the next billion devices with wi-fi. arXiv preprint arXiv:1505.06815, 2015.

[36] V. Talla, S. Pellerano, H. Xu, A. Ravi, and Y. Palaskas. Wi-fi rf energy harvesting for battery-free wearable radio platforms. In RFID (RFID), 2015 IEEE International Conference on, pages 47-54. IEEE, 2015.

[37] N. Tesla. My Inventions: The Autobiography of Nikola Tesla. Hart Bros., 1982.

[38] M. S. Trotter and G. D. Durgin. Survey of range improvement of commercial rfid tags with power optimized waveforms. In IEEE RFID 2010.

[39] M. S. Trotter, J. D. Griffin, and G. D. Durgin. Power-optimized waveforms for improving the range and reliability of rfid systems. In IEEE RFID 2009.

[40] C. Valenta and G. Durgin. Harvesting wireless power: Survey of energy-harvester conversion efficiency in far-field, wireless power transfer systems. IEEE Microwave Magazine, 2014.

[41] H. Visser, A. Reniers, and J. Theeuwes. Ambient rf energy scavenging: Gsm and wlan power density measurements. In EuMC 2008.

[42] B. Waters, A. Sample, P. Bonde, and J. Smith. Powering a ventricular assist device (vad) with the free-range resonant electrical energy delivery (free-d) system. Proceedings of the IEEE 2012. 